\title{
Aprovechamiento y transformación sostenible de materias primas vegetales de uso artesanal en el departamento del Chocó, Colombia
}

\section{Advantage and sustainable transformation of vegetal raw materials of artisan use in the department of Chocó, Colombia}

\section{Angélica Asprilla*}

\section{RESUMEN}

La documentación del conocimiento tradicional de uso, manejo y aprovechamiento de las especies de werregue y damagua en el departamento del Chocó, es el principal objetivo del presente estudio. Se realizó un análisis químico de las especies nativas con potencial tintóreo en uso por las comunidades afrocolombianas e indígenas del Chocó, mediante su sensibilización y adiestramiento con prácticas de extracción de materias primas artesanales amigables con el ambiente. Para el logro de los objetivos del proyecto se desarrollaron entrevistas etnobotánicas y talleres de intercambio y convalidación de información, continuando con la evaluación rápida de la oferta natural del recurso, consistente en inventariar y analizar el estado fitosanitario de todos y cada uno de los individuos presentes en una parcela de $100 \mathrm{~m} x 100 \mathrm{~m}$ y el desarrollo de capacitaciones a los artesanos Se logró documentar el conocimiento tradicional de cinco especies tintóreas y cuatro de fibra artesanal, determinando un potencial muy alto de productos (45 con diseños y utilidades surtidos) elaborados por los indígenas del Litoral del San Juan y el Alto Baudó. Finalmente se sensibiliza y capacita a 128 líderes y artesanos de la zona.

Palabras clave: Conocimiento tradicional; Etnobotánica; Artesanías; Damagua; Werregue.

\begin{abstract}
The documentation of the traditional knowledge of use, handling and advantage of the species of werregue and damagua in the department of Choco, is the main objective of the present study. A chemical analysis of the native species with tintoreo potential was realised in use by the afrocolombianas and indigenous communities of Chocó, by means of their sensitization and training with practices of extraction of friendly artisan raw materials with the atmosphere. For the profit of the objectives of project etnobotánicas interviews were developed and factories of interchange and confirmation of information, continuing with the fast evaluation of the natural supply of the resource, consisting of inventorying and analyzing the phytosanitary state of each and every one of the individual presents in a parcel of $100 \mathrm{~m} \times 100 \mathrm{~m}$ and the development of qualifications to the craftsmen were managed to document of the traditional knowledge of five tintóreas species and four fibers artisan, determining a very high product potential (45 with assorted designs and utilities) made by the natives of the Coast of San Juan and the Baudo Stop. Finally it is sensitized and enable to 128 leaders and craftsmen of the zone.
\end{abstract}

Keywords: Traditional knowledge; Etnobotánico; Crafts; Damagua; Werregue.
* Bióloga, Especialista en proyectos de desarrollo, Directora Fundación EXISTIR, Quibdó, Colombia. e-mail: angelicap71@yahoo.es Recibido: Julio 30, 2009 Aceptado: Agosto 13, 2009 


\section{Bioetnia Volumen 6 № 2 (julio-diciembre), 2009}

\section{INTRODUCCIÓN}

En Colombia las plantas han jugado un papel importante, sirviéndole al hombre como medicina, alimento, en la construcción de viviendas, mobiliarios, en la fabricación de telas, tintes, aceites, esencias, en instrumentos de caza, forraje y otras materias primas de uso artesanal. El Chocó es considerada una de las regiones más diversas en especies vegetales de gran importancia cultural y socioeconómica, que han utilizado a través del tiempo sus pobladores para suplir sus múltiples necesidades alimenticias, medicinales y mágicoreligiosas entre otras; en este sentido, a nivel regional y local se han realizado trabajos dirigidos a conocer las plantas y el uso que las diferentes comunidades hacen de este recurso. En la costa Pacífica vale la pena resaltar la investigación realizada por Galeano (2000), quien afirma que los usos más importantes involucran la extracción selectiva de madera para la construcción, leña y material para aplicaciones tecnológicas. Pino et al. (2003), reportaron que las comunidades afro descendientes de Quibdó utilizan el recurso flora en la medicina, alimentación, construcción y actividades mágico-religiosas, entre otras.

Las materias primas de uso artesanal en las últimas décadas han venido jugando un papel importante, puesto que cada vez más familias se vinculan al desarrollo de la actividad artesanal. En dicho contexto y en el marco de las funciones otorgadas por Ley al Ministerio de Ambiente, Vivienda y Desarrollo Territorial, y en convenio con la Fundación Aid To Artisans, y con la posterior participación de la Embajada Real de los Países Bajos, el Instituto de Investigaciones Biológicas Alexander von Humboldt y el Instituto de Investigaciones Ambientales del Pacífico (IIAP), se realizó el «diagnóstico inicial sobre manejoin situ y protocolos de aprovechamiento de productos no maderables del bosque (werregue, damagua, tagua, iraca y paja blanca)» y los «protocolos de aprovechamiento in situ para las especies de uso artesanal werregue, damagua, tagua y paja blanca en Chocó y Boyacá». En el marco de estos trabajos se identificó que la tagua es un importante recurso natural no maderable disponible en varias regiones del país, pero especialmente en el occidente de Boyacá, que tiene gran potencial de aprovechamiento por características de la especie como buena capacidad de regeneración natural y alto volumen de producción, además de ser la semilla de tagua, un material que permite la elaboración de una amplia gama de productos artesanales, con una creciente demanda en el mercado nacional e internacional. Con el convenio de cooperación interinstitucional con el Ministerio de Ambiente, Vivienda y Desarrollo Territorial para impulsar la adopción de buenas prácticas de aprovechamiento sostenible de las materias primas silvestres, promover la permanencia de los oficios y agregar valor al producto, Artesanías de Colombia S.A. y el Ministerio de Ambiente, Vivienda y Desa- rrollo Territorial firmaron el convenio 037 de 2007 con el objeto de «desarrollar e implementar un programa de apoyo técnico, ambiental y empresarial, con el fin de fomentar el uso y manejo sostenible de los recursos naturales y otras materias primas artesanales, en el marco del Plan Estratégico de Mercados Verdes». Ello permitirá avanzar en el desarrollo de una artesanía cuya riqueza en diseño armonice con el respeto al medio ambiente, facilitando así su acceso al mercado internacional.

El desarrollo del convenio concibe la generación de alianzas interinstitucionales con corporaciones, institutos de investigación, gobernaciones, municipios y ONG, para cubrir el trabajo con especies prioritarias del sector artesanal que presentan problemas de sostenibilidad o de ilegalidad en el aprovechamiento. En este sentido se pretende incorporar al desarrollo del convenio, el trabajo con la tagua, werregue, iraca, damagua y cabecinegro en las regiones naturales del departamento de Chocó. Entre los principales problemas que enfrenta el sector artesanal se resaltan los siguientes: de acuerdo con la información suministrada por CODECHOCÓ, pocos artesanos cuentan con permisos de aprovechamiento para la recolección de la tagua, werregue, iraca, damagua y cabecinegro, y se asume que en la generalidad los recolectores $\mathrm{y}$ artesanos trabajan con estos recursos de origen presuntamente ilegal. Muchos artesanos y comercializadores de productos en tagua, werregue, iraca, damagua y cabecinegro, han perdido clientes internacionales por la dificultad que tienen para obtener permisos de aprovechamiento, porque no existen las condiciones para demostrar la trazabilidad de los productos a lo largo de la cadena productiva.

La comercialización de los productos a la fecha se encuentra desarticulada porque no garantiza que la comunidad perciba mejores ingresos. Por esta razón el proyecto se traza como objetivo en esta oportunidad, cuantificar la oferta natural de werregue y la damagua principalmente, iraca, cabecinegro y tagua, esperando aportar información básica para planificar el uso racional y sostenible del recurso.

\section{ÁREA DE ESTUDIO}

Zonas de muestreo para el desarrollo del proyecto. El municipio de Quibdó es la capital del departamento del Chocó situado en el occidente de Colombia, en la región de la llanura del Pacífico. La ciudad se encuentra a orillas del río Atrato, uno de los principales afluentes del país y una de las zonas con más alta pluviosidad del mundo. Cuenta con una población de 105.172 habitantes, distribuidos en seis comunas. Quibdó se encuentra localizado a los $5^{\circ} 41^{\prime}$ norte y $76^{\circ} 40^{\prime}$ oeste. Presenta una temperatura promedio de $27^{\circ} \mathrm{C}$ y una humedad relativa de $85 \%$, la precipitación oscila entre 4.000-8.000 mm anuales y presenta las zonas de vida bosque pluvial tropical (bp-P) y bosque muy húmedo tropical (bmh-T) (Espinal 1997).

El municipio del Alto Baudó, está situado en la región de 


\section{Tabla 1}

Comunidades de socialización

Comunidad

Consejo Comunitario de Pie de Pató o Ciudad Baudó

Cabildo Indígena Bella luz

Consejo Comunitario de Winandó

Consejo Comunitario de Tutunendo

Consejo Comunitario de Opogodó

Consejo Comunitario de Cabecera

Consejo Comunitario de Malaguita

Consejo Comunitario de Pángala

Cabildo Indígena Unión Guaimía

Cabildo Indígena Estrella Pángal

Consejo Comunitario de Docordó

Consejo Comunitario de Palestina

Cabildo Indígena Chagpien Tordó

Cabildo Indígena Durrab dur

Cabildo Indígena San Bernardo

Cabildo Indígena Burujón

Cabildo Indígena Unión san Juan

Cabildo Indígena Balsalito

Cabildo Indígena Chagpien Medio

\section{Municipio habitacional}

Alto Baudó

Quibdó

Condoto

Buenaventura

Litoral del San Juan las calmas ecuatoriales y según el sistema de Holdrigue, corresponde a las zonas de vida de los bosques muy húmedos tropical (bmh-t) y bosque pluvial tropical (bpt). El primero se caracteriza por temperaturas mayores de $24^{\circ} \mathrm{C}$ y precipitaciones entre 4.000-8.000 $\mathrm{mm}$, y el segundo por temperatura mayor a $24^{\circ} \mathrm{C}$ y precipitación superior a $8.000 \mathrm{~mm}$ anuales. El clima de la zona se encuentra determinado por vientos marítimos que circulan del océano hacia el continente. Su cabecera municipal es Pie de Pato, que se encuentra a $5^{\circ} 31^{\prime} 33^{\prime \prime}$ de latitud norte y 76 $99^{\prime} 44^{\prime \prime}$ de longitud oeste, respeto al meridiano de Greenwinch; la superficie territorial del municipio es de 1.532 $\mathrm{km}^{2}$, altura de $50 \mathrm{msnm}$, temperatura promedio $28^{\circ} \mathrm{C}$. El municipio se enmarca dentro de la cuenca del río Baudó y como contexto geográfico se ubica en el centro y sur del departamento del Chocó, limitando al norte con los municipios de Quibdó, Bojayá y Bahía Solano; al oriente con los municipios de Quibdó, Río Quito y Cantón de San Pablo; al occidente con el municipio de Nuquí; al sur con los municipios de Bajo Baudó y Medio Baudó. El municipio del Alto Baudó fue segregado del municipio del Baudó, que tenía por capital a Pizarro, por medio de la Ordenanza $\mathrm{N}^{\circ} 16$ del 25 de noviembre de 1958 y creado como municipio el 1 de enero de 1959. Su población mayoritariamente de origen negro (73.3\%) seguida por una alta presencia de la etnia indígena (26.6\%) que habita en resguardos legalmente constituidos. Ubicación y localización geográfica: su cabecera Pie de Pató, está localizada en la margen izquierda del río Baudó, a los 05³1'33' de latitud norte y 76 $59^{\prime} 42^{\prime \prime}$ de longitud oeste. Su altura sobre el nivel del mar es de $50 \mathrm{~m}$. Temperatura media de $28^{\circ} \mathrm{C}$, precipitación media anual es de $6.439 \mathrm{~mm}$ y dista de Quibdó $80 \mathrm{~km}$. La cabecera municipal Pie de Pató, fue conocida antiguamente como Puerto Yacup y a la fecha se llama Ciudad Baudó. El municipio cuenta con los siguientes consejos comunitarios: Amparraido, Apartadó, Chachajo, Nauca, San Francisco de Cugucho, Santa Catalina, Playita (Río Catrú), Yucal, Batatal, Bellavista, Chigorodo, Santa Rita, Puerto Córdoba, Puerto Echeverri, Puerto Martínez y Puerto Alegre, y los resguardos indígenas de Agua Clara, Bella Luz, Río Amporá, Puerto Alegre, La Divisa, Río Catrú y Dubasa, Río Jurubida-Chori y Alto Baudó, Dominico Londoño, Apartadó, Puerto Libia Tripicay (Tabla 1).

El municipio del Litoral de San Juan, ubicado en el departamento del Chocó fundado en 1993, segregado del municipio de Istmina. Su extensión es de $3.755 \mathrm{~km}^{2}$ y cuenta con una temperatura promedio de $28^{\circ} \mathrm{C}$. Se encuentra a $25 \mathrm{msnm}$. Cuenta con una población de 850 habitantes en el sector 


\section{Bioetnia Volumen 6 № 2 (julio-diciembre), 2009}

urbano y el sector rural 5.891 habitantes. Sus tierras se distribuyen en los pisos térmicos cálido, templado y frío.

\section{MÉTODOS}

En la presente investigación se contemplaron los siguientes procesos y procedimientos:

La información etnobotánica de las especie evaluadas, se obtuvo de los datos almacenados en la base de datos Spica del Centro de Documentación del IIAP, diversas investigaciones etnobotánicas realizadas en la misma (antecedentes), además de testimonios obtenidos con algunos poseedores del conocimiento tradicional sobre el uso de las plantas artesanales y tintóreas.

Las visitas a la comunidad se realizaron en forma continua, durante cuatro meses, con el fin de conocer e intercambiar la cosmovisión de las comunidades afrodescendientes e indígenas chocoanas.

El desarrollo del trabajo en campo se realizó de la siguiente manera:

a. Socialización de la propuesta con los miembros de las comunidades, que se desarrolló por medio de talleres y presentaciones orales, donde se generó un espacio de discusión y aprobación del desarrollo de la propuesta, lo que permitió que la comunidad participara activamente; asimismo se acordó cuáles conocedores locales participarían en el trabajo de campo. Con anterioridad se realizó contacto con los miembros del consejo comunitario y autoridades locales.

b. La técnica utilizada para el diálogo con los artesanos se realizó mediante visitas directas, charlas y entrevistas. En este proceso con la comunidad se trabajó de manera individual y grupal, donde los miembros de un sector, tenían la oportunidad de escuchar a su vecino y a veces hasta corregirles algún tipo de información, lo que creó mayor ambiente de confiabilidad y credibilidad en la recepción de la información; adicionalmente se recogieron testimonios, vivencias y antecedentes sobre la forma de uso de las especies artesanales y tintóreas. También se realizaron 16 talleres comunitarios con intensidad de dos días por zona a lo largo del proyecto, en los que en primera instancia se sensibilizó y se impartieron los fundamentos teóricos de extracción de materias primas artesanales amigables con el ambiente. Posteriormente se procedió a socializar la información que se obtuvo en las entrevistas personalizadas convalidando con ello la información recopilada y analizada previamente; finalmente se amplía el conocimiento de las especies tintóreas con el grueso de la comunidad asistente.

c. Para la recolección del material vegetal se escogieron los sitios indicados por los artesanos y se realizaron nueve salidas exploratorias de campo con intensidad de un día, en las que se demostró y evaluó el aprendizaje, habilidades y destrezas para el aprovechamiento sostenible de las materias primas artesanales en estudio. Acto seguido se cuantificó la oferta natural de werregue y damagua, que consiste en trazar una parcela de una hectárea y dentro de ésta registrar todos y cada uno de los individuos de las especies en cuestión. Una vez en la zona, los investigadores registran en una matriz básica de datos las características fundamentales y relevantes de cada uno de los individuos seleccionados, georreferenciando el sitio exacto de la colección de la especie con su respectivo registro fotográfico, estado del ciclo biológico del individuo, frecuencia de aprovechamiento, partes aprovechadas, fin de aprovechamiento, forma de utilización e ingresos generados por aprovechamiento del bien, entre otras, como estrategia de validación de la información que se capturó en las entrevistas personalizadas.

d. Trabajo de herbario para la determinación taxonómica de los individuos debidamente preservados en prensa con alcohol al 70\%, fue necesario el traslado de las colecciones al laboratorio de botánica de la Universidad Tecnológica del Chocó «Diego Luis Córdoba» utilizando claves taxonómicas de Gentry (1993) y Mahecha (1997) entre otras, y la confrontación de especímenes del herbario Chocó.

e. Talleres comunitarios de evaluación de actividades y generación de correctivos a la problemática. Se reunió nuevamente la comunidad y se expusieron las experiencias y/o moralejas de cada uno de los asistentes frente a los conocimientos teóricos impartidos y las prácticas en campo, a través del estímulo de un debate con preguntas semiestructuradas.

f. La información socioeconómica se analizó con estadística descriptiva, a través de la estimación de la media aritmética y el porcentaje de la misma. Finalmente la información se presentó mediante tablas, figuras y gráficos según requerimiento de cada resultado.

\section{RESULTADOS Y DISCUSIÓN}

Mediante socialización se presentó el proyecto con la participación de la organización étnico territorial ACIVA, que facilitó el desarrollo del proyecto con la articulación entre cabildos indígenas de la zona, participando así tanto los cabildos afiliados como los que no lo están. Para el caso de las comunidades afrocolombianas, se llevó a cabo el proceso de articulación entre los consejos comunitarios y el IIAPArtesanías de Colombia, el Consejo Comunitario Mayor de Docordó, que lidera la sensibilización local de sus homólogos para participar en el proyecto una vez es sensibilizado por los funcionarios del IIAP-Artesanías de Colombia. También se socializó el proyecto en 21 comunidades étnicas, distribuidas 


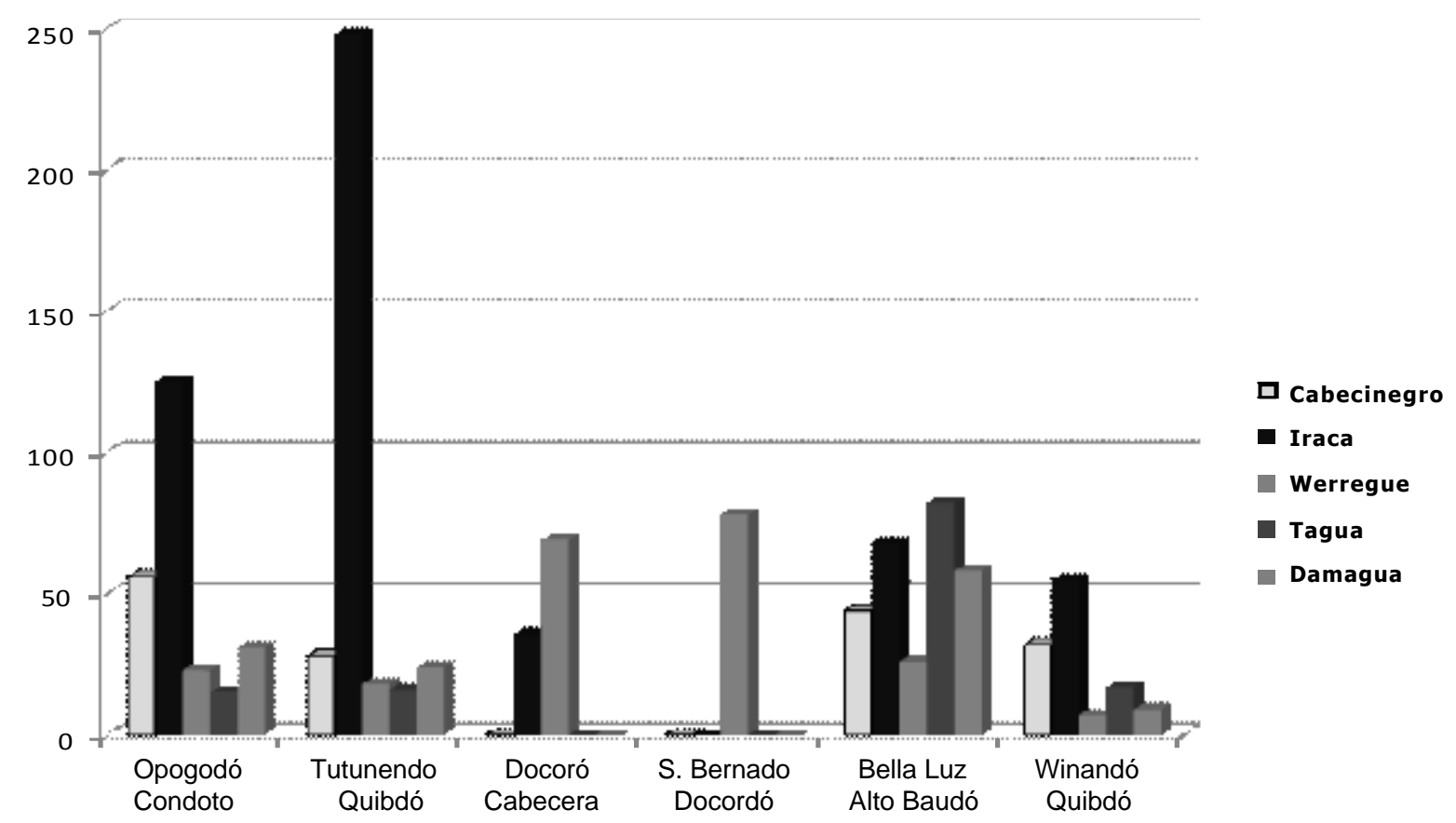

Gráfica 1. Número de individuos por especie en una hectárea de bosque de los municipios de Quibdó, Condoto, Alto Baudó, Litoral del San Juan y Buenaventura.

en 13 cabildos indígenas y ocho consejos comunitarios. De los 13 cabildos indígenas, nueve pertenecen al municipio del Litoral del San Juan-Docordó, dos al municipio de Quibdó y uno al municipio del Alto Baudó-alcaldía municipal Baudó o Pie de Pató. De los ocho consejos comunitarios, uno corresponde al municipio de Quibdó, uno al municipio del Alto Baudó, uno al municipio de Condoto, dos al municipio de Buenaventura y dos al Litoral del San Juan (Tabla 1). Finalmente se logró la socialización y desarrollo del proyecto en cinco municipios y dos departamentos (Chocó y Valle del Cauca).

Desarrollo de expediciones de campo para la cuantificación de oferta natural de werregue, damagua, tagua, iraca, cabecinegro. De las cinco especies estudiadas en la aproximación de su oferta natural en algunas zonas del Chocó y parte del Valle del Cauca, se destaca el werregue, con presencia en todos los puntos de muestreo; la damagua en cuatro, la iraca en cinco, tagua y cabecinegro en cuatro (Gráfica 1).

Damagua. La comunidad de Winandó, está registrada como un área potencial de oferta natural de damagua en la base de datos de Mercados Verdes de CODECHOCÓ, que debe ser reestructurada frente a los valores de oferta natural del presente estudio (9 individuos/ha), con la gravedad de que el estudio se realizó en un bosque que la comunidad seleccionó como área con mayor presencia de damagua e intervención agrícola bastante considerable. Por otro lado, los resultados de las entrevistas personalizadas y talleres comunitarios de intercambio y convalidación de información con la comunidad, permitieron determinar que la comunidad no es extractora, ni artesana de damagua y que en los casos en que se requiere la materia prima se encarga a comunidades indígenas de procedencia del Baudó, pues existen contactos estratégicos (caminos) entre las zonas del territorio. Winandó, es una comunidad que se desempeña principalmente en la agricultura y aspira a avanzar en esta actividad, aunque existe un pequeño grupo de mujeres artesanas con tejidos de hilo apalancadas por las madres fami de Bienestar Familiar, y otro pequeño grupo que diseña objetos artesanales con iraca, entre otras especies, para la movilización de sus productos agrícolas. Tutunendo con oferta de 24 individuos/ha, se muestra como un punto potencial, con el atenuante de que la comunidad, a pesar de manejar las técnicas de extracción, en la actualidad no presenta interés de aprovechamiento del recurso, puesto que se desempeñan en el ecoturismo, agricultura y minería artesanal principalmente. Opogodó con 31 individuos, se posiciona en segundo punto con potencial de extracción, con el atenuante de que la comunidad en el momento no tiene interés de aprovechamiento del recurso, por la poca preparación de los dos grupos de artesanos del lugar con técnicas atractivas de transformación de la materia prima y el agravante que los bosques de menor intervención antrópica donde se registró el potencial de especies está siendo colonizado con pasos agigantados por la actividad 


\section{Bioetnia Volumen 6 № 2 (julio-diciembre), 2009}

minera con retroescabadora, bombas, dragas y motobombas. Bella Luz Indio con 51 individuos/ha y las otras comunidades registradas para el Alto Baudó, son en realidad los puntos de extracción del recurso damagua en la actualidad, actividad realizada principalmente por los indígenas y en segundo lugar por los afrocolombianos, quienes se destacan en el acopio y la comercialización intermunicipal de la fibra natural, a demás de desarrollar la extracción por un grupo de hombres de la zona. Se reconoce el grupo de Cugucho como extractores y comercializadores de damagua del Baudó y el grupo de extractores de Bella Luz Indio, quienes requieren apoyo en diversos sentidos para garantizar la sostenibilidad de la especie.

Para unárea de muestreo de 0.1 ha la distribución diamétrica de mayor importancia para la damagua fue los latizales con 58 individuos, distribuidos en 36 individuos para Bella Luz, 13 para Tutunendo y nueve para Opogodó, seguido por el diámetro de fustal, repartidos entre 15 para Opogodó, 11 para Tutunendo, cuatro para Bella Luz y tres para Winandó; finalmente el diámetro brinzal con 31 individuos, de los cuales 18 se registraron para Bella Luz, siete para Opogodó, seis para Winandó y Tutunendo no presentó.

En el contexto anterior, se propone a Bella Luz en el Alto Baudó y Opogodó en Condoto como un área que debe establecerse como zona de recuperación de la damagua, para cuidar los individuos juveniles que garantizarán la supervivencia de la especie en el Chocó y los municipios, máxime cuando ésta se encuentra registrada en la lista roja de especies con alta vulnerabilidad antrópica y apego cultural de uso. A Bella Luz Indio, Tutunendo en Quibdó y Opogodó se consideran como centros de monitoreo de la especie y principalmente a Bella Luz y las otras zonas de extracción de damagua en el Alto Baudó, puesto que es la distribución que tradicionalmente las comunidades utilizan para aprovechar la especie, dada la facilidad de manejo de la corteza de los árboles con el agravante que a esa fecha los individuos de la especie no ha generado descendencia. Aquí es donde deben centrarse los controles de líderes comunitarios, los consejos comunitarios, cabildos indígenas, CODECHOCÓ, el IIAP, las ONG y toda institucionalidad que procure la sostenibilidad de la especie. Opogodó, Tutunendo y Bella Luz Indio, se proponen como centros de implementación de parcelas permanentes productoras de semillas de damagua y otras investigaciones biológicas con la finalidad de garantizar la producción de germoplasmas de la especie y fomentar la recuperación y conservación de la especie.

Werregue. A pesar de tener registro en todos los puntos de muestreo de influencia directa del proyecto, se destaca con los mayores valores en Docordó, Baudó y Condoto. La especie del werregue presenta escasez en las comunidades de los resguardos del oriente (Santa María de Pangala Taparal, Chagpien y Tordó), mientras que aumenta en las comunida- des del sur y el norte, en las comunidades de los resguardos (Nuevo Pitalito, Papayo, Unión San Bernardo, Buena Vista, Tiocilirio, Puerto Pizario, Docordó Unión Balsalito, San Antonio de Togoromá y Río Pichimá), sin perder de vista la demanda imperiosa de la materia prima para las familias artesanas de la zona que son $94.3 \%$ aproximadamente del total de familias del territorio. Además del desbalance de individuos registrados entre comunidades para abastecerse y proveer a otras comunidades como el caso de las comunidades del oriente, quienes deben ser abastecidas por algunas comunidades del sur, norte y del vecino municipio del Medio San Juan- Andagoya, a donde todavía no se ha llegado con proyectos como éste. Cabe resaltar que San Bernardo, Burujón, Palestina, Cabecera, Malaguita y Docordó cabecera municipal, tienen una atractiva oferta natural de la especies, y se debe continuar con su sensibilización y capacitación sobre prácticas de aprovechamiento sostenible, e implementación de asesorías educativas sobre aprovechamiento racional y efectivo de éste y otros recursos del medio.

Otras especies. Opogodó en Condoto, se destaca con un buen potencial de especies de cabecinegro, seguido por Quibdó en Tutunendo, San Isidro, Río Quito, entre otros. Respecto de la especie se identifica un agravante representado en el aprovechamiento de la espata, porque $67.3 \%$ de la comunidad que aprovecha la especie compromete la fructificación, puesto que se corta toda la inflorescencia. Tutunendo, seguido por Opogodó, Bella Luz Indio y Winandó en cuarto lugar, presentan el mayor potencial de oferta natural de Iraca. Esta especie es altamente potencial para su aprovechamiento puesto que las prácticas utilizadas en la actualidad no comprometen significativamente la reproducción de la planta, además que la alta capacidad adaptativa del individuo al medio garantiza la permanencia de la especie.

Acuerdos y/o compromisos interinstitucionales generados al final del proyecto. Como resultado de los talleres comunitarios de evaluación de actividades y generación de correctivos a la problemática de las materias primas de uso artesanal por los asistentes a las diferentes actividades en las distintas comunidades se destacan las premisas:

\section{Desde lo local}

1. En el futuro inmediato, concertar precios justos entre las comunidades poseedoras de materias primas artesanales y las que no las poseen, y garantizar la calidad del recurso.

2. Conformar por comunidad un grupo de personas conocedoras de las especies maderables con potencial artesanal, para identificar árboles madres que estén produciendo semillas y hacerles seguimiento, pensando en el establecimiento de bancos de semillas para la recuperación y conservación de la especie.

3. Las comunidades indígenas y afrocolombianas interesadas en hacer un verdadero aprovechamiento sostenible de sus recursos y legalizar su actividad artesanal con 
materias primas de la localidad, se comprometen a definir el tipo de permiso que requieren y a realizar una carta de solicitud de permisos de extracción de materias primas y registro de movilización de productos para su debida comercialización a CODECHOCÓ.

4. Los representantes legales, presidentes o persona autorizada por la comunidad, certificará los resultados del proceso, frente a la lectura semestral de la información.

5. Hacer el debido acompañamiento a los instructores para la formulación de los proyectos de implementación de resultados del presente proyecto.

6. Recibir las herramientas media luna correspondientes a la disponibilidad del recurso y el número de familias dedicadas a la actividad en la zona para garantizar la sostenibilidad del recurso.

7. Los comunicadores, líderes, artesanos y extractores de la materia prima sensibilizada y capacitada en el manejo de las prácticas sostenibles y herramientas, se comprometen a replicar en sus lugares de origen y donde se requiera con el fin de conservar la especie.

\section{Institucionales}

1. Formular y desarrollar en el futuro los proyectos por comunidades de acuerdo con sus necesidades.

2. Artesanías de Colombia y el IIAP avanzarán en la investigación para adaptar varas metálicas a las medialunas entregadas en esta oportunidad, en pro de garantizar su uso pora facilitar el trabajo y reducir la tasa de corta de varas para el aprovechamiento del recurso artesanal.

3. El IIAP apoyará el proceso de la gestión de los permisos de aprovechamiento de materias primas y movilización de productos artesanales de las comunidades, buscando ante CODECHO la disminución de los costos.

4. Artesanías de Colombia y el IIAP avanzarán en la consolidación del documento final sobre la palma del werregue y se enviará a la comunidad de San Bernardo para su debida traducción en lengua y consolidar la publicación de una cartilla bilingüe entre las partes.

\section{CONCLUSIONES}

El departamento del Chocó y parte del Valle del Cauca registran una considerable diversidad de especies artesanales y en gran parte del territorio hay escasez de oferta de dichas materias primas. A la fecha las principales comunidades proveedoras de materias primas (werregue y damagua) de uso artesanal son Nohanamá, quebrada Macedonia, Docordó, quebrada del municipio del Medio San Juan-Andagoya y Docordó, cabecera municipal del municipio del Litoral del San Juan. El gremio de las palmas constituye el grupo con mayor número de especies con potenciales para la elaboración de artesanías.

Las comunidades de Pángala, Unión Guaimia, Estrella
Pángal, Taparalito, San José, Chagpien Tordó, Chagpien Medio, Durrab presentan escasez de materias primas como el werregue. Mientras que la situación mejora en Nuevo Pitalito, Papayo, Guarataco, San Bernardo, Burujón, Buenavista, Tiocilirio, Unión San Juan, Balsalito, Togoromá, Loma linda, Pichima, Palestina, Malaguita, Cabecera, Carrá y Docordó sin que ello signifique que no hay problema de abastecimiento de materias primas de uso artesanal. El municipio del Baudó se declara como centro de abastecimiento de fibra natural de damagua, por su abundancia y abastecimiento de pedidos de diferentes zonas de la región.

\section{RECOMENDACIONES}

Para la comunidad y sus líderes, avanzar en:

- Dar cumplimiento a los acuerdos alcanzados en el marco del proyecto.

- Perfeccionar y diversificar la cadena y productos artesanales.

- Los artesanos deben gestionar el posicionamiento de los productos en nichos de mercados regional e internacional, consolidar el local y ampliar el nacional.

- Avanzar en el perfeccionamiento de los tintes naturales de materias primas artesanales de apego cultural en la zona. Para las instituciones ambientales y de desarrollo de la región:

- CODECHOCÓ, debe actualizar su base de datos de artesanos, extractores de materias primas y procedencia de dichos recursos.

- CODECHOCÓ, debe promover y facilitar los permisos de extracción de materias primas de uso artesanal y registros de movilización de productos.

- CODECHOCÓ, debe hacer seguimiento, monitoreo y control a los permisos de aprovechamiento de materias primas en el territorio.

- EL IIAP, las universidades, las ONG y otras instituciones con objetivos afines, deben avanzar en el proceso investigativo de las especies proveedoras de materias primas artesanales.

- Artesanías de Colombia y toda la institución con razón misional generadoras de desarrollo comunitarios y fomento de productos artesanales, deben promover y apoyar la implementación de proyectos direccionados a solucionar y/o mitigar los resultados problema identificados en el presente proyecto.

\section{LITERATURA CITADA}

Bernal, R., G. G. Galeano. 1993. Palmas del Andén Pacífico. En: Leyva, P. (ed). Colombia Pacífico. Tomo I. Bogotá: Editorial del Fondo FEN, Instituto de Ciencias Naturales, Universidad Nacional de Colombia. p. 220-31,

Calderón, E. 2000. Lista preliminar de plantas en vía de extinción. Bogotá, DC: 


\section{Bioetnia Volumen 6 No 2 (julio-diciembre), 2009}

Instituto de Investigaciones de Recursos Biológicos Alexander Von Humboldt.

Forero, E, Gentry, A. 1989. Lista anotada de plantas del departamento del Chocó. Bogotá, DC: Instituto de Ciencias Naturales. Museo de Historia Natural. p. 50-4.

Galeano, G., R. Bernal. 2004. Catálogo de espermatofitos en el Chocó Biogeográfico. Familia Arecaceae. En: Rangel-CH, J.O. (ed). Colombia diversidad biótica IV: El Chocó biogeográfico/costa pacífica. Instituto de Ciencias Naturales Universidad Nacional de Colombia. p. 135-48.
Galeano, G. 1992. Patrones de distribución de las palmas de Colombia. Caldasia. 21 (2): 599-607.

Gentry, A. H. 1993. Riqueza de especies y composición florística de la comunidad de plantas de la región del Chocó: una actualización Colombia Pacífico. Tomo I. Proyecto biopacífico Colombia, Bogotá, DC: Fondo FEN; p. 2019

Mahecha, G. 1997. Fundamentos y metodología para la identificación de las plantas. Bogotá, DC: Ministerio de Medio Ambiente proyecto Biopacífico, Instituto Humboldt. p. 282 